\title{
REVIEWS
}

\section{Knocking down barriers: advances in siRNA delivery}

Kathryn A. Whitehead*, Robert Langer*‡ and Daniel G. Anderson ${ }^{\ddagger}$

Abstract | In the 10 years that have passed since the Nobel prize-winning discovery of RNA interference (RNAi), billions of dollars have been invested in the therapeutic application of gene silencing in humans. Today, there are promising data from ongoing clinical trials for the treatment of age-related macular degeneration and respiratory syncytial virus. Despite these early successes, however, the widespread use of RNAi therapeutics for disease prevention and treatment requires the development of clinically suitable, safe and effective drug delivery vehicles. Here, we provide an update on the progress of RNAi therapeutics and highlight novel synthetic materials for the encapsulation and intracellular delivery of nucleic acids.

\section{RNA interference} (RNAi). A fundamental pathway in eukaryotic cells by which a short piece of RNA is able to induce the destruction of mRNA containing a complementary sequence.

Small interfering RNA (siRNA). RNA fragments approximately $21-23$ nucleotides long that are capable of inducing the sequence-specific destruction of complementary mRNA.

RNA-induced silencing complex

(RISC). The protein complex responsible for the binding and unwinding of the double-stranded siRNA within the cytoplasm, and for the subsequent identification and destruction of the target mRNA.

* Department of Chemical Engineering, ${ }^{\ddagger}$ The David H. Koch Institute for Integrated Cancer Research, Massachusetts Institute of Technology, Cambridge, Massachusetts 02142, USA.

Correspondence to D.G.A. e-mail:dgander@mit.edu doi: $10.1038 / n r d 2742$
RNA interference (RNAi) gained international attention in 1998 when Fire, Mello and colleagues discovered the ability of double-stranded RNA to silence gene expression in the nematode worm Caenorhabditis elegans ${ }^{1}$. Three years later, Tuschl and co-workers published their celebrated proof-of-principle experiment demonstrating that synthetic small interfering RNA (siRNA) could achieve sequence-specific gene knockdown in a mammalian cell line $^{2}$. The first successful use of siRNA for gene silencing in mice was achieved for a hepatitis $\mathrm{C}$ target shortly thereafter ${ }^{3}$. Since that time, the biotechnology sector has made considerable efforts in the advancement of siRNA therapeutics for the treatment of various disease targets, including viral infections $s^{4,5}$ and cancer ${ }^{6-8}$.

RNAi is a fundamental pathway in eukaryotic cells by which sequence-specific siRNA is able to target and cleave complementary mRNA ${ }^{2}$. RNAi is triggered by the presence of long pieces of double-stranded RNA, which are cleaved into the fragments known as siRNA (21-23 nucleotides long) by the enzyme Dicer ${ }^{9}$. In practice, siRNA can be synthetically produced and then directly introduced into the cell, thus circumventing Dicer mechanics (FIG. 1). This shortcut reduces the potential for an innate immune interferon response and the shutdown of cellular protein expression that can occur following the interaction of long pieces ( $>30$ nucleotides) of doublestranded RNA with intracellular RNA receptors ${ }^{10}$.

Once siRNA is present in the cytoplasm of the cell, it is incorporated into a protein complex called the RNAinduced silencing complex (RISC) ${ }^{11}$. Argonaute 2, a multifunctional protein contained within RISC, unwinds the siRNA, after which the sense strand (or passenger strand) of the siRNA is cleaved ${ }^{12}$. The activated RISC, which contains the antisense strand (or guide strand) of the siRNA, selectively seeks out and degrades mRNA that is complementary to the antisense strand ${ }^{13}$ (FIG. 1). The cleavage of mRNA occurs at a position between nucleotides 10 and 11 on the complementary antisense strand, relative to the $5^{\prime}$-end ${ }^{14}$. The activated RISC complex can then move on to destroy additional mRNA targets, which further propagates gene silencing ${ }^{15}$. This extra potency ensures a therapeutic effect for 3-7 days in rapidly dividing cells, and for several weeks in non-dividing cells ${ }^{16}$. Eventually, siRNAs are diluted below a certain therapeutic threshold or degraded within the cell, and so repeated administration is necessary to achieve a persistent effect.

Theoretically, when using appropriately designed siRNA, the RNAi machinery can be exploited to silence nearly any gene in the body, giving it a broader therapeutic potential than typical small-molecule drugs. Indeed, it has already been reported that synthetic siRNAs are capable of knocking down targets in various diseases in vivo, including hypercholesterolaemia ${ }^{17}$, liver cirrhosis $^{18}$, hepatitis B virus (HBV) $)^{4,19}$, human papillomavirus $^{20}$, ovarian cancer ${ }^{21}$ and bone cancer ${ }^{22}$. In order for these advances to be implemented in a clinical setting, safe and effective delivery systems must be developed. While 'naked', chemically modified siRNA has shown efficacy in certain physiological settings such as the brain ${ }^{23}$ and the lung ${ }^{24}$, there are many tissues in the body that require an additional delivery system to facilitate transfection. This is because naked siRNA is subject to degradation by endogenous enzymes, and is too large $(\sim 13 \mathrm{kDa})$ and too negatively charged to cross cellular membranes. 


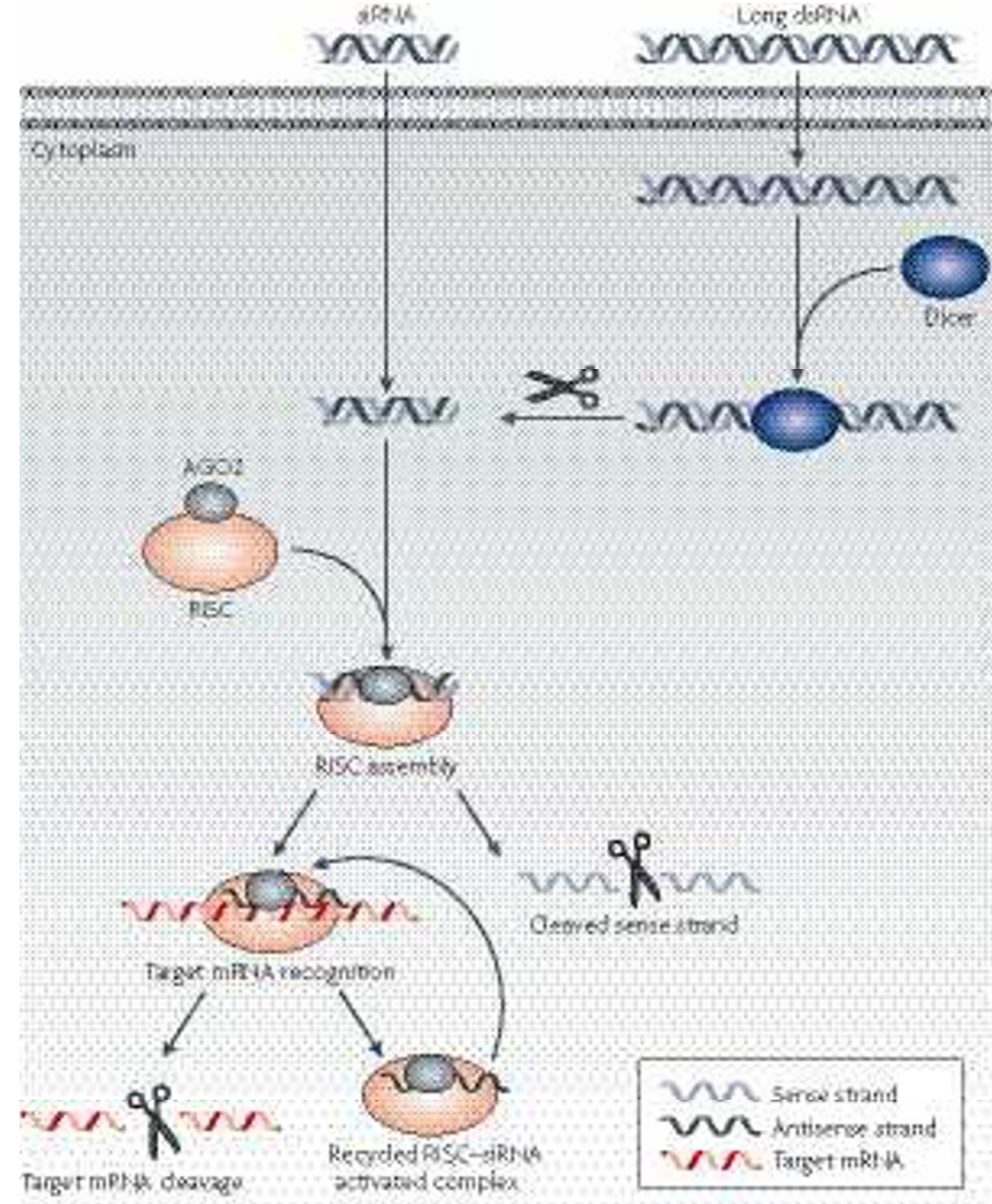

Figure 1 | The mechanism of RNA interference. Long double-stranded RNA (dsRNA) is introduced into the cytoplasm, where it is cleaved into small interfering RNA (siRNA) by the enzyme Dicer. Alternatively, siRNA can be introduced directly into the cell. The siRNA is then incorporated into the RNA-induced silencing complex (RISC), resulting in the cleavage of the sense strand of RNA by argonaute 2 (AGO2). The activated RISC-siRNA complex seeks out, binds to and degrades complementary mRNA, which leads to the silencing of the target gene. The activated RISC-siRNA complex can then be recycled for the destruction of identical mRNA targets.

Antisense strand

The strand of the siRNA

molecule that is

complementary to the target

mRNA, which activates RISC

and has an important role in

target mRNA identification

and destruction.

Transfection

The process of delivering nucleic acid material into the cell.
The issue of effective and non-toxic delivery is a key challenge and serves as the most significant barrier between siRNA technology and its therapeutic application.

\section{Modes of siRNA administration}

The ease of siRNA delivery is partly dependent on the accessibility of the target organ or tissue within the body. Localized siRNA delivery - that is, application of siRNA therapy directly to the target tissue - offers several benefits, including the potential for both higher bioavailability given the proximity to the target tissue, and reduced adverse effects typically associated with systemic administration. By contrast, systemic delivery, meaning the intravenous injection of delivery particles that then travel throughout the body to the target organ or tissue, requires that particles have the ability to avoid uptake and clearance by non-target tissues (FIG. 2).
There are several tissues that are amenable to topical or localized therapy, including the eye, skin, mucus membranes, and local tumours ${ }^{25-28}$ (TABLE 1). Local siRNA delivery is particularly well-suited for the treatment of lung diseases and infections. The direct instillation of siRNA into the lung through intranasal or intratracheal routes enables direct contact with lung epithelial cells. These cells play a part in a myriad of lung conditions and infections, including cystic fibrosis, asthma, influenza and the common cold ${ }^{24}$. It has been reported that respiratory syncytial virus (RSV) replication can be inhibited by nasally administered siRNA formulated with or without transfection agents in mice ${ }^{29,30}$. Progress in the treatment of RSV continues with Phase II clinical trials using an aerosolized siRNA delivery system $^{31}$. Intratracheal administration of siRNA has also been reported to offer prophylactic and therapeutic effects in the treatment of severe acute respiratory syndrome ${ }^{32}$.

Another example of local delivery is direct intratumoral injection of siRNA delivery complexes into various mouse xenograft models. siRNA complexed with the delivery agent polyethyleneimine (PEI) was shown to inhibit tumour growth upon intratumoral injection in mice bearing glioblastoma xenographs ${ }^{28}$. Niu and co-workers have also reported naked siRNA efficacy upon direct injection into a subcutaneous cervical cancer model in mice ${ }^{20}$.

\section{Barriers to systemic siRNA delivery in vivo}

In contrast to the direct accessibility of localized targets, many tissues can only be reached through the systemic administration of delivery agents in the bloodstream. siRNA formulations for systemic application face a series of hurdles in vivo before reaching the cytoplasm of the target cell (FIG. 2). Post-injection, the siRNA complex must navigate the circulatory system of the body while avoiding kidney filtration, uptake by phagocytes, aggregation with serum proteins, and enzymatic degradation by endogenous nucleases ${ }^{33}$.

Phagocytosis serves as a significant immunological barrier, not only in the bloodstream but also in the extracellular matrix of tissues. Phagocytic cells such as macrophages and monocytes remove foreign material from the body to protect against infection by viruses, bacteria and fungi. Unfortunately, phagocytes are also highly efficient at removing certain therapeutic nanocomplexes and macromolecules from the body, and steps must be taken to avoid opsonization when designing drug delivery vehicles ${ }^{33}$.

Egress from the bloodstream and across the vascular endothelial barrier poses a significant challenge for delivery of siRNA to many tissues within the body. In general, molecules larger than $5 \mathrm{~nm}$ in diameter do not readily cross the capillary endothelium, and therefore will remain in the circulation until they are cleared from the body. There are certain tissues, however, that allow the entry of larger molecules, including the liver, spleen, and some tumours. These organs allow the passage of molecules up to $200 \mathrm{~nm}$ in diameter, which can accommodate a typical drug delivery nanocarrier ${ }^{34}$. 


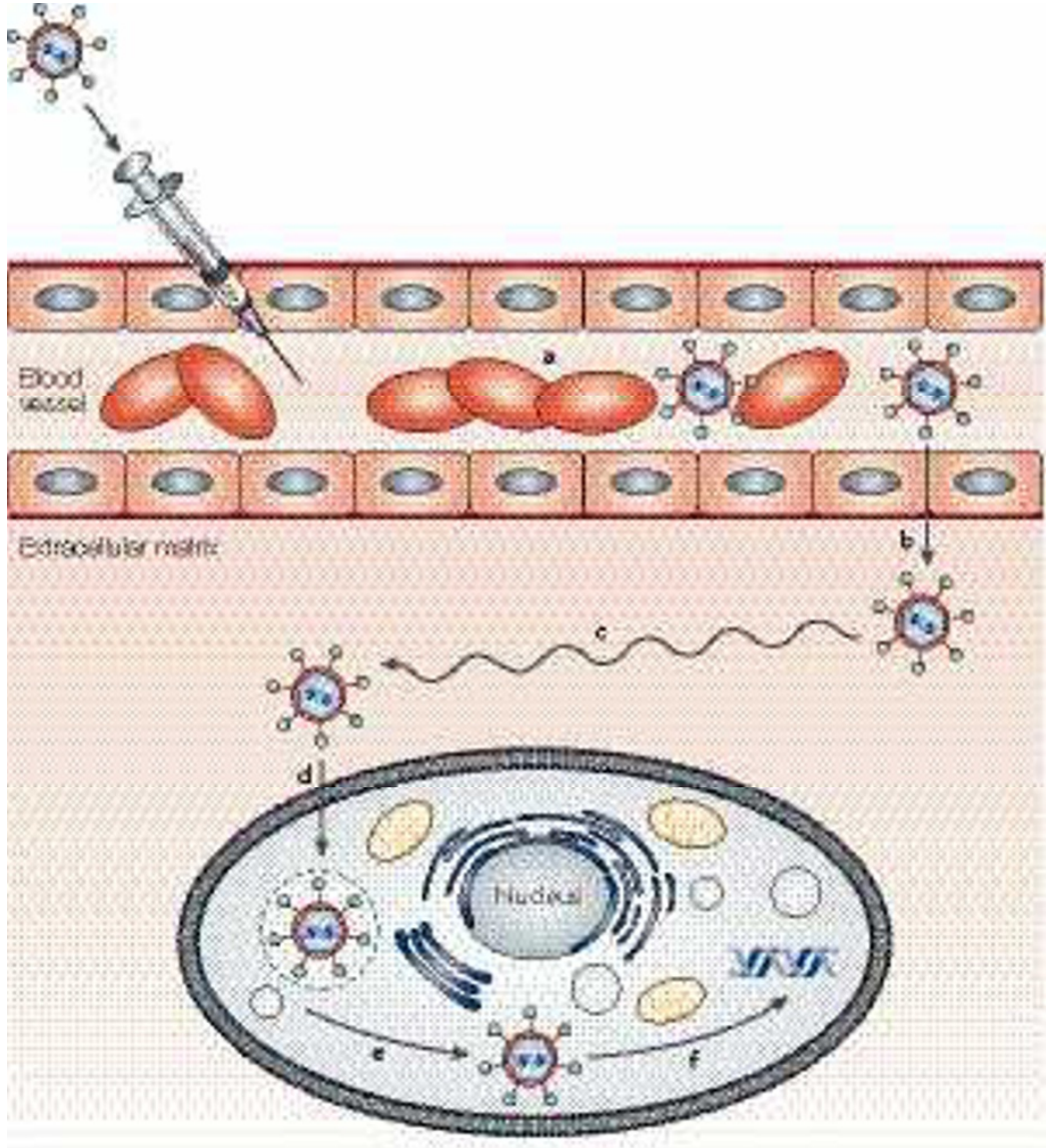

Figure 2 | Physiological barriers to the systemic delivery of small interfering RNA (siRNA) nanoparticles. An injected nanoparticle must avoid filtration, phagocytosis and degradation in the bloodstream (a); be transported across the vascular endothelial barrier (b); diffuse through the extracellular matrix (c); be taken up into the cell (d); escape the endosome (e); and unpackage and release the siRNA to the RNA interference (RNAi) machinery (f).
siRNAs can induce nonspecific activation of the immune system through the Toll-like receptor 7 (TLR7) pathway ${ }^{38,39}$. This effect can be reduced by the incorporation of 2'-O-methyl modifications into the sugar structure of selected nucleotides within both the sense and antisense strands ${ }^{38,40}$ (FIG. 3a). 2'-O-methyl modifications have also been shown to confer resistance to endonuclease activity ${ }^{41}$ and to abrogate off-target effects when incorporated into the seed region, which corresponds to nucleotides 2-8 on the antisense strand ${ }^{42}$. Other common modification approaches to mitigate enzymatic degradation include the introduction of phosphorothioate backbone linkages at the 3 '-end of the RNA strands to reduce susceptibility to exonucleases. It is also possible to incorporate alternative 2 ' sugar modifications (for example, a fluorine substitution) to increase resistance to endonucleases ${ }^{43}$.

Another strategy to improve the therapeutic efficacy of siRNA involves the conjugation of small molecules or peptides to the sense strand of the siRNA. Several small molecules have been reported to increase target-gene knockdown in vitro, including membrane-permeant peptides $^{44}$ and polyethylene glycol (PEG) ${ }^{45}$. Of particular note are cholesterol-modified siRNAs, which have demonstrated increased binding to serum albumin, resulting in improved biodistribution to certain targets including the liver (FIG. 3b). Cholesterol-modified siRNA were capable of silencing apolipoprotein B (ApoB) targets in mouse liver and jejunum, and of ultimately reducing total cholesterol levels ${ }^{46}$. Another study by DiFiglia and co-workers details the ability of a cholesterol-modified siRNA to knockdown a gene associated with Huntington's disease. A single intrastriatal injection was able to delay the abnormal behavioural phenotype observed in a rapid-onset mouse model of this disease ${ }^{23}$.

Given the success of cholesterol-modified siRNA in vivo, Wolfrum and co-workers attempted to identify alternative lipid-like molecules to serve as RNA conjugates for improved delivery of siRNA ${ }^{47}$. Specifically, fatty acids and bile-salt derivatives were conjugated to siRNA and injected into mice and hamsters in order to elucidate how modified siRNA conjugates interact with the high-density lipoprotein (HDL) and low-density lipoprotein (LDL) receptors that enable delivery to the liver. It was found that shorter fatty-acid chain lengths $\left(<\mathrm{C}_{18}\right)$ did not induce gene knockdown, whereas bilesalt derivatives and fatty-acid conjugates with longer chain lengths enabled potent silencing in hepatocytes via the HDL receptor ${ }^{47}$.

Another example of the possibility of introducing beneficial modifications to nucleic acid therapeutics comes from the antisense drug mipomersen. Mipomersen is a $2^{\prime}-O$-(2-methoxyethyl)-modified singlestranded RNA molecule that is targeted to $\mathrm{ApoB}^{48}$, a protein that has been implicated in cardiovascular disease. Isis Pharmaceuticals has reported promising Phase II safety and efficacy results, and a Phase III trial to assess efficacy in patients with a familial history of hypercholesterolaemia is currently underway in a joint venture with Genzyme ${ }^{49}$. 


\section{Table 1 | Modes of siRNA delivery and potential targets}

\begin{tabular}{|c|c|c|c|}
\hline $\begin{array}{l}\text { Mode of } \\
\text { administration }\end{array}$ & Potential organ target & Potential disease target & Refs \\
\hline \multirow[t]{4}{*}{ Topical } & Eye & Macular degeneration & 102 \\
\hline & Skin & Atopic dermatitis & 25 \\
\hline & Vagina & Herpes simplex virus & 27 \\
\hline & Rectum & Inflammatory bowel disease & 105 \\
\hline \multirow[t]{4}{*}{ Local/direct } & Lung & SARS & 32 \\
\hline & Brain & Huntington's disease & 23 \\
\hline & Spinal cord & Chronic pain & 90 \\
\hline & Isolated tumour & Glioblastoma multiforme & 28 \\
\hline \multirow[t]{4}{*}{ Systemic } & Liver & Hypercholesterolaemia & 57 \\
\hline & Heart & Myocardial infarction & 106 \\
\hline & Kidney & Kidney disease & 61 \\
\hline & Metastasized tumours & Ewing's sarcoma & 97 \\
\hline
\end{tabular}

SARS, severe acute respiratory syndrome; siRNA, small interfering RNA.

\section{Properties of synthetic delivery nanoparticles}

For tissues and cells that are not amenable to the delivery of naked or chemically modified siRNA, delivery of nanoparticles that incorporate siRNA are used. In general, delivery vehicles are designed to both facilitate uptake into the target tissue of interest and, when used for systemic delivery, to protect siRNA payloads and inhibit nonspecific delivery. Below, we highlight several important characteristics of delivery nanoparticles and provide specific examples of their construction and use.

Surface properties. The surface charge of a delivery nanoparticle can significantly influence the way it interacts with the target cell and other physiological molecules. In the simplified in vitro setting, a positively charged delivery vehicle can facilitate uptake by associating with the negatively charged cellular membrane ${ }^{50}$. A positive charge also promotes complex formation and compression with the polyanionic nucleic acids of the siRNA. The situation, however, becomes more complicated in vivo as negatively charged serum proteins in the bloodstream will often bind to a positively charged nanocomplex, therefore rendering it ineffective. The addition of PEG or other hydrophilic conjugates to the surface of a delivery vehicle can assist in mitigating this problem ${ }^{51}$. Additionally, PEG conjugation can control particle size and prevent particle aggregation in the presence of serum ${ }^{51}$.

Coating of the nanoparticle with hydrophilic molecules such as PEG can also play an important role in the ability of the siRNA delivery carrier to evade the immune system and associated phagocytes. PEG forms a barrier around nanoparticles that provides steric stabilization and protection from the physiological surroundings ${ }^{52}$. The length of the PEG chain can have a significant influence on its stabilization and protective properties, and chain length is typically optimized for each individual delivery system ${ }^{53,54}$.
Biodistribution. The biodistribution of siRNA can be significantly influenced through formulation with a delivery vehicle. Systemic administration of synthetic delivery nanoparticles often results in accumulation in the organs of the reticuloendothelial system, including the liver, spleen, kidneys and lungs ${ }^{16,55,56}$. It is no coincidence that much of the successful siRNA delivery seen in recent years has targeted disease within these organs $\mathbf{s}^{4,29,30,57,58}$.

Excretion through the kidney typically occurs for molecules less than $50 \mathrm{kDa}$ in size ${ }^{59}$. As such, naked siRNA experiences rapid renal clearance upon systemic administration ${ }^{43}$. Several studies monitored the biodistribution of siRNA in mice after an intravenous injection and observed naked siRNA accumulation in the kidney and urine within 5 minutes of administration ${ }^{60,61}$. By complexing siRNA with synthetic materials, the size of the delivery nanoparticle can be increased to avoid glomerular filtration through the kidneys and reserve the siRNA for alternative organ targets ${ }^{59}$.

Additionally, it has been reported that certain siRNA formulations are capable of accumulation in subcutaneous tumours ${ }^{22,62}$. This phenomenon has been attributed to the enhanced permeability and retention (EPR) effect. It has therefore become a common approach to exploit the leaky vasculature of tumours for the purposes of directed delivery ${ }^{7,62,63}$. Others have also reported success in targeting tumours through conjugation to ligands such as antibodies ${ }^{64,65}$.

Toxicity. Even the most efficacious siRNA delivery agents are rendered useless if they provoke unacceptable toxicity on either a cellular or systemic level. Viral vectors, which were among the first vehicles to be studied for siRNA delivery, can induce unacceptable levels of toxicity through the activation of immune responses ${ }^{66}$. Therefore, synthetic lipids and polymers have been developed to offer alternatives to viral vectors for nucleic acid delivery applications, and are carefully formulated to avoid stimulation of the immune system ${ }^{39}$. Clearance of larger molecular mass materials typically requires them to be biodegradable. The use of biodegradable, high molecular mass polycations and polymers containing linkages that can be cleaved inside the cell can help reduce cytotoxicity ${ }^{67}$.

\section{Synthetic materials for siRNA delivery}

Synthetic materials have demonstrated potential as effective non-viral siRNA delivery carriers. Although many types of compound have been investigated as potential candidates, this Review focuses on synthetic materials that have successfully delivered nucleic acids in vivo. An overview of the delivery agents presented here can be found in TABLE 2.

Liposomes and lipid-like materials. Unilamellar and multilamellar liposomes are commonly used as pharmaceutical delivery vehicles ${ }^{68}$. In an aqueous environment, certain materials have the ability to form liposomes, in which a lipid bilayer forms a sphere with an aqueous core. For example, one set of polar head-groups can create the 


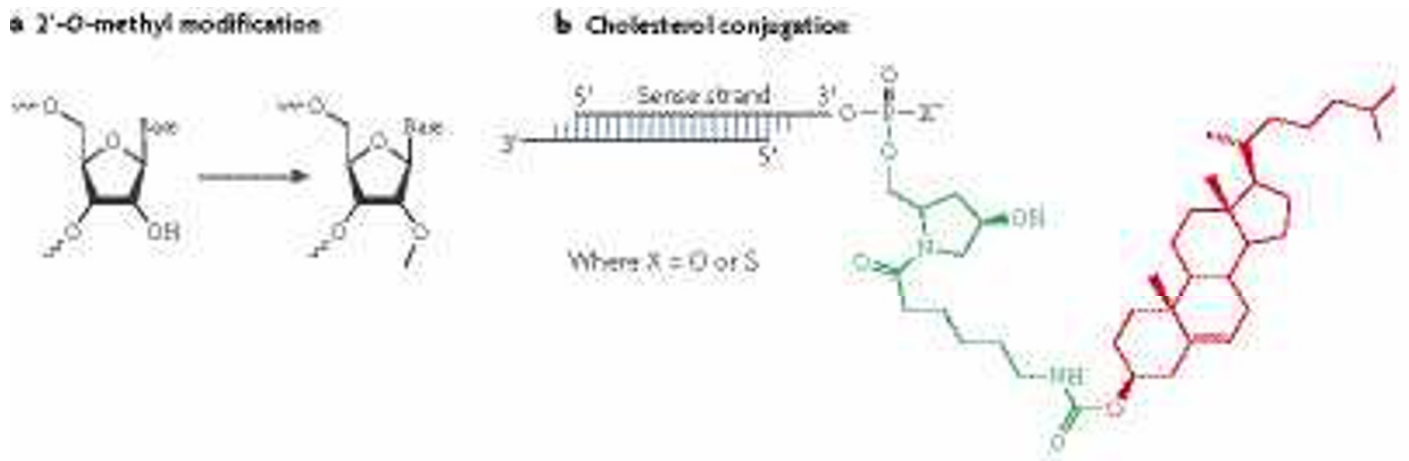

Figure 3 | Two common small interfering RNA (siRNA) modifications used for therapeutic applications. a | The 2'-O-methyl sugar modification prevents activation of the Toll-like receptor 7 immune response and confers enzymatic resistance to the siRNA molecule. $\mathbf{b}$ | The conjugation of cholesterol to the sense strand of an siRNA duplex improves delivery of naked siRNA to certain cellular targets, including hepatocytes. The cholesterol conjugate is shown in red, and the linker is shown in green.

outer surface of the nanocomplex, while another set of polar head-groups faces the interior hydrophilic core, which houses the nucleic acid payload ${ }^{69}$ (FIG. 4). It is also possible for liposomes to be amorphous in structure, with the lipids and nucleic acids interspersed throughout. Liposomes can be created using single or multiple types of lipid, which allows for additional flexibility when optimizing the physical and chemical properties of the nanoparticle ${ }^{68}$.

Liposomes have been used for the delivery of nucleic acids for over 20 years, originating with studies by Felgner and colleagues detailing the ability of the cationic lipid DOTMA ( $N$-[1-(2,3-dioleyloxy)propyl]- $N, N, N$ trimethlylammonium chloride) to deliver both DNA and RNA into mouse, rat and human cell lines ${ }^{70,71}$. More recently, stable nucleic acid-lipid particle (SNALP) formulations have demonstrated efficacy in several models in vivo. A study by Morrissey and co-workers indicated that HBV replication was inhibited through the delivery of an siRNA-SNALP complex that targeted HBV RNA. Three daily intravenous injections of $3 \mathrm{mg}$ per $\mathrm{kg}$ per day reduced serum HBV levels by at least one order of magnitude, and the effect was specific, dose-dependent and lasted for up to 7 days after dosing ${ }^{4}$. In addition, Zimmerman and colleagues demonstrated the ability of SNALPs to enable knockdown of ApoB in the liver of cynomolgus monkeys ${ }^{57}$. A single siRNA injection resulted in dose-dependent silencing of ApoB mRNA expression in the liver 48 hours after administration, with maximal silencing of more than $90 \%$. Knockdown was confirmed to be caused by ApoB mRNA cleavage at precisely the site predicted for the RNAi mechanism, and persisted for 11 days at the highest administered dose of $2.5 \mathrm{mg}$ per $\mathrm{kg}^{57}$.

An additional study details the use of SNALP formulations to combat the Zaire strain of Ebola virus, which proves fatal to $90 \%$ of its victims via haemorrhagic fever ${ }^{72}$. When contained within the SNALP formulation, siRNA targeting of the polymerase gene of the virus completely protected guinea pigs against viraemia and death when administered shortly after an Ebola virus challenge. It is important to note that preliminary studies in mice have indicated an activation of interferon $\alpha$ and $\beta$, suggesting that stimulation of the immune system may also have a role in efficacy ${ }^{72}$.

More recently, Sato and co-workers used vitaminA-coupled Lipotrust liposomes to deliver anti-gp46 siRNA to fibrogenic hepatic cells for the treatment of liver cirrhosis. Five administrations of the siRNA lipocomplexes were reported to resolve liver fibrosis and prolong survival in rats that had otherwise lethal liver cirrhosis, in a doseand duration-dependent manner. It was also shown that rescue was not related to off-target effects or associated with recruitment of the innate immune system ${ }^{18}$.

The use of lipid complexes for localized siRNA administration has also proved beneficial. Vaginal instillation of cationic liposomes comprised of Oligofectamine and siRNA targeting herpes simplex virus (HSV)-2 reportedly led to uptake by the epithelial and lamina propria cells in the vagina in mice and protected against lethal infection for up to 9 days. The siRNA complexes protected mice when administered before and/or after (otherwise) lethal HSV-2 challenge ${ }^{27}$. In another study, intrathecal administration of complexes formulated by i-FECT and siRNA protected mice from fatal Japanese encephalitis virus and West Nile virus after intracranial administration, and reduced pain receptor expression following intrathecal administration in rats $^{73}$.

Also of note are several synthetic lipid-based materials that have demonstrated DNA transfection efficiency. Similar materials may also potentially serve as candidates for siRNA delivery, given the similarities involved in the two types of nucleic acid delivery ${ }^{74}$. For example, Wheeler et al. used liposomes comprising a combination of the cationic lipid GAP-DLRIE ( $N$-(3-aminopropyl)$N, N$-dimethyl-2,3-bis(dodecyloxy)-1-propanaminium bromide) and the neutral co-lipid DOPE (dioleoylphosphatidylethanolamine) to transfect mouse lung with a gene that confers chloramphenicol resistance. After a single intranasal administration, gene expression was enhanced by more than 100-fold relative to plasmid DNA alone, followed by a gradual return to baseline levels by 21 days post-administration ${ }^{75}$. Also of interest are transactivating transcriptional activator (TAT)-modified 


\begin{tabular}{|c|c|c|c|c|c|}
\hline Material & Model & Target & Route & Animal & Refs \\
\hline \multicolumn{6}{|c|}{ Liposomes and lipids } \\
\hline i-FECT & $\begin{array}{l}\text { Japanese encephalitis } \\
\text { virus (JEV) and West } \\
\text { Nile virus (WNV) }\end{array}$ & $\begin{array}{l}\text { JEV and WNV } \\
\text { envelope }\end{array}$ & Intracranial & Mouse & 70 \\
\hline \multirow[t]{4}{*}{ Lipidoids } & Dyslipidaemia & FVII/ApoB & Intravenous & $\begin{array}{l}\text { Mouse, rat, } \\
\text { monkey }\end{array}$ & 30 \\
\hline & Dyslipidaemia & FVII/ApoB & Intravenous & $\begin{array}{l}\text { Mouse, } \\
\text { hamster }\end{array}$ & 79 \\
\hline & Malaria & Haem oxygenase 1 & Intravenous & Mouse & 80 \\
\hline & Hypercholesterolaemia & PCSK9 & Intravenous & Mouse, rat & 17 \\
\hline LipoTrust & Liver cirrhosis & gp46 & Intravenous & Rat & 18 \\
\hline Oligofectamine & $\begin{array}{l}\text { Herpes simplex virus } 2 \\
\text { (HSV-2) }\end{array}$ & $\begin{array}{l}\text { HSV-2-associated } \\
\text { viral proteins UL27 } \\
\text { and UL29 }\end{array}$ & Intravaginal & Mouse & 27 \\
\hline \multirow[t]{3}{*}{ SNALP } & Hepatitis B virus (HBV) & HBV & Intravenous & Mouse & 4 \\
\hline & Dyslipidaemia & ApoB & Intravenous & Monkey & 56 \\
\hline & Ebola (Zaire) & Polymerase L & Intravenous & Guinea pig & 69 \\
\hline \multicolumn{6}{|c|}{ Cationic polymers } \\
\hline \multirow[t]{2}{*}{ Cyclodextrin } & $\begin{array}{l}\text { Ewing's sarcoma tumour } \\
\text { xenograft }\end{array}$ & EWS-FLI1 & Intravenous & Mouse & 94 \\
\hline & Healthy monkey model & RRM2 & Intravenous & Monkey & 95 \\
\hline $\begin{array}{l}\text { Dynamic } \\
\text { PolyConjugate }\end{array}$ & Dyslipidaemia & ApoB/PPARa & Intravenous & Mouse & 98 \\
\hline \multirow{4}{*}{$\begin{array}{l}\text { Poly- } \\
\text { ethyleneimine }\end{array}$} & Glioblastoma xenograft & PTN & Intratumoral & Mouse & 28 \\
\hline & Formalin-induced pain & NMDAR2B & Intrathecal & Rat & 87 \\
\hline & Cervical tumour xenograft & HPV E6/E7 & Intratumoral & Mouse & 20 \\
\hline & Ovarian tumour xenograft & HER2 & Intraperitoneal & Mouse & 88 \\
\hline \multicolumn{6}{|c|}{ Small interfering RNA (siRNA) conjugates } \\
\hline \multirow[t]{2}{*}{ Cholesterol } & Dyslipidaemia & ApoB & Intravenous & Mouse & 46 \\
\hline & Huntington's disease & Huntingtin gene & Intrastriatal & Mouse & 23 \\
\hline $\begin{array}{l}\text { Fatty acids/ } \\
\text { bile salts }\end{array}$ & Dyslipidaemia & ApoB & Intravenous & $\begin{array}{l}\text { Mouse, } \\
\text { hamster }\end{array}$ & 47 \\
\hline
\end{tabular}

ApoB, apolipoprotein B; EWS-FLI1, Ewing's sarcoma-friend leukaemia virus integration 1; FVII, factor VII blood protein; gp46, a glycoprotein gene; HER2, human epidermal growth factor receptor 2; HPV E6/E7, human papillomavirus oncogenes; NMDAR2B, $\mathrm{N}$-methyl-D-aspartate receptor type 2B; PCSK9, proprotein convertase subtilisin/kexin type 9; PPARa, peroxisome proliferatoractivated receptor $\alpha$; PTN, pleiotrophin (a secreted growth factor); RRM2, ribonucleoside-diphosphate reductase subunit M2; SNALP, stable nucleic acid-lipid particles.

liposomes, which, when complexed with a gene encoding green fluorescence protein (GFP) and injected locally, induced the expression of GFP in Lewis lung carcinoma tumour cells in mice ${ }^{50}$.

Although liposomes are among the most popular nucleic acid delivery agents, some concerns regarding their safety for therapeutic use remain. Toxicity of certain cationic lipid particles has been reported both in vitro and in vivo ${ }^{76-78}$, and certain synthetic agents have been found to induce a gene signature of their own that might increase the off-target effects of siRNA ${ }^{79,80}$. Despite these issues, liposomes show promise for future clinical use, as evidenced by the approval of PEGylated liposomes for doxorubicin and amphotericin B delivery by the US Food and Drug Administration ${ }^{79,81}$.
In another study, a combinatorial library of lipid-like materials was developed for use in siRNA delivery ${ }^{30}$. These 'lipidoids' were synthesized through the conjugate addition of alkyl-acrylates and -amides to primary and secondary amines and then studied in cell culture. One leading candidate for in vivo gene knockdown was identified as $98 \mathrm{~N}_{12}-5(1)$, which comprises five 12-carbon alkyl-acrylamide chains attached to an amine core (FIG. 4d). Formulations of this material with siRNA were capable of achieving potent and persistent silencing of various lung and liver targets in mice, rats and cynomolgus monkeys ${ }^{30}$.

In a more recent primate study, $98 \mathrm{~N}_{12}-5(1)$ was also used to deliver siRNA against PCSK9, a protein that regulates LDL receptor protein levels and function ${ }^{17}$. Liver-specific siRNA silencing of PCSK9 reduced PCSK9 

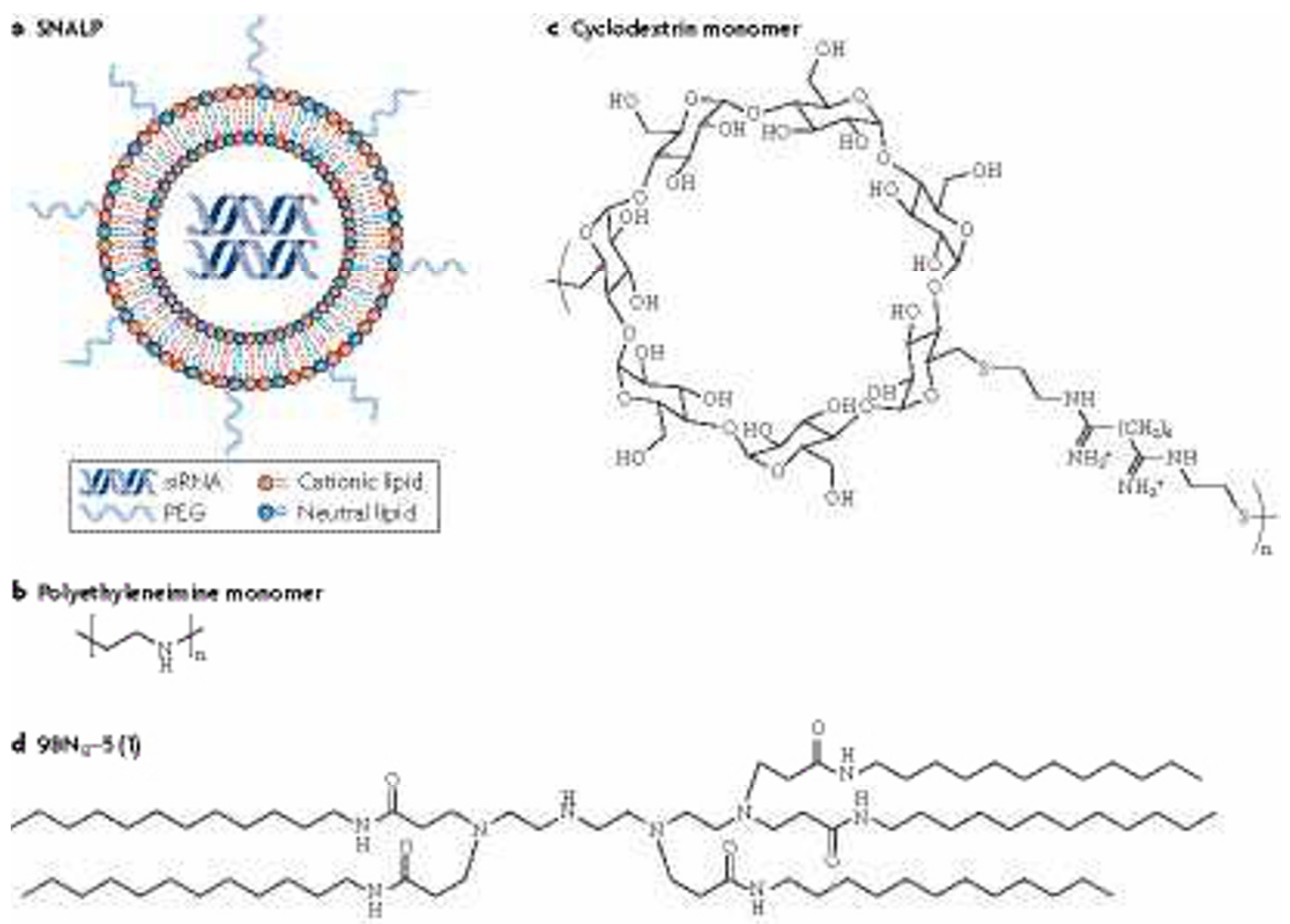

Figure 4 | Synthetic materials for small interfering RNA (siRNA) delivery. a | A representation of stable nucleic acid-lipid particles (SNALPs), which are liposomes comprising cationic lipids, non-ionic lipids and polyethylene glycol (PEG). siRNA is contained in the hydrophilic interior of the particle. $\mathbf{b} \mid$ Polyethyleneimine is used to fabricate both linear and branched polymeric delivery agents. c | A cyclodextrin-based delivery agent. d | The lipidoid $98 \mathrm{~N}_{12}-5(1)$.

mRNA levels by $50-70 \%$ in mice and rats, as well as reducing human PCSK9 transcript levels in transgenic mice by more than $70 \%$. Silencing persisted for up to 3 weeks after a single intravenous dose, indicating that anti-PCSK9-lipidoid complexes may serve as a potent and effective treatment for hypercholesterolaemia ${ }^{17}$. Importantly, lipidoid materials were shown to facilitate siRNA delivery without disrupting endogenous microRNA processing ${ }^{82}$. Finally, Hmox1, a gene expressed in the liver encoding the protein haem oxygenase 1 , has also been silenced in mice by siRNA nanoparticles formulated with lipidoids. Knockdown of Hmoxl may represent a potential approach for the treatment of malaria infection and disease progression ${ }^{83}$.

Polymers. Cationic polymers with a linear or branched structure can serve as efficient transfection agents because of their ability to bind and condense large nucleic acids into stabilized nanoparticles ${ }^{84,85}$. Such materials have also been shown to stimulate nonspecific endocytosis as well as endosomal escape $\mathrm{e}^{79}$. A proposed mechanism for this is the 'proton-sponge' effect ${ }^{84}$, whereby buffering of the endosome leads to an accumulation of ions within this compartment and an osmotic pressure that eventually bursts the endosome ${ }^{86,87}$.

PEI is a broadly investigated delivery carrier for the administration of a wide range of nucleotide-based therapies, including DNA, siRNA and oligonucleotides ${ }^{88,89}$.
It has also been frequently used for various local siRNA delivery applications. Intrathecal administration of PEIsiRNA complexes was reported to selectively knockdown a pain receptor in rats. Maximal effect occurred on day 3 for mRNA levels and day 7 for associated protein levels following an injection of $5 \mathrm{mg}$ of siRNA targeting a subunit of the NMDA ( $N$-methyl-D-aspartate) receptor $\mathrm{NR}_{2} \mathrm{~B}^{90}$. PEI has also shown efficacy in a subcutaneous mouse tumour model. The intraperitoneal administration of complexed siRNA led to the delivery of the intact siRNA into the tumours and a marked reduction of tumour growth through siRNA-mediated downregulation of human epidermal growth factor 2 (HER2; also known as ERBB2 $)^{91}$.There has been significant concern regarding the toxicity of PEI at higher molecular masses and high doses $^{92,93}$. However, strategies to modify the structure of PEI to reduce toxicity while retaining its potent ability to transfect cells are in development ${ }^{94-96}$.

Cyclodextrin polymers have also been developed as siRNA delivery agents. Tumour growth in a mouse model of metastatic Ewing's sarcoma was shown to be inhibited by the systemic delivery of nanoparticles formed by cyclodextrin, the targeting ligand transferrin, and siRNA specific for the EWS-FLI1 fusion gene commonly associated with the condition. Knockdown was not observed upon removal of the targeting ligand, nor was there any evidence of immune stimulation or toxicity ${ }^{97}$. In another study, Heidel and co-workers used 


\begin{tabular}{|c|c|c|c|}
\hline Company & Disease & $\begin{array}{l}\text { Mode of } \\
\text { administration }\end{array}$ & Status \\
\hline Allergan & $\begin{array}{l}\text { Age-related macular } \\
\text { degeneration }\end{array}$ & Topical & Phase II \\
\hline Alnylam & $\begin{array}{l}\text { Respiratory syncytial } \\
\text { virus }\end{array}$ & Local/direct & Phase II \\
\hline Nucleonics & Hepatitis B virus & Systemic & Phase I \\
\hline $\begin{array}{l}\text { Quark Pharmaceuticals/ } \\
\text { Pfizer }\end{array}$ & Acute renal failure & Systemic & Phase I \\
\hline Opko Health & $\begin{array}{l}\text { Age-related macular } \\
\text { degeneration }\end{array}$ & Topical & Phase III \\
\hline Silence/Quark/Pfizer & $\begin{array}{l}\text { Diabetic macular } \\
\text { oedema }\end{array}$ & Topical & Phase II \\
\hline Transderm & $\begin{array}{l}\text { Pachyonychia } \\
\text { congenita }\end{array}$ & Topical & Phase la/b \\
\hline
\end{tabular}

siRNA, small interfering RNA.
(VEGFR), has shown therapeutic potential in its inhibition of the excessive vascularization of the eye that leads to $\mathrm{AMD}^{26}$.

In 2004, the first clinical trial involving siRNA was carried out by Acuity Pharmaceuticals for the treatment of AMD. The completed Phase II trials reported that all doses were well tolerated with a lack of adverse systemic effects. Testing has now moved into Phase III trials, which have been taken over by Opko Health. Allergan is currently conducting a Phase II clinical trial on an siRNA for AMD, with completed Phase I results indicating minimal side effects and improved vision in some of the patients. Although Silence Therapeutics also had an siRNA product for AMD in the pipeline, they have now refocused their Phase II clinical study on the treatment of diabetic macular oedema, which is another condition caused by leaky vasculature within the eye. Importantly, a recent study has reported that anti-VEGF siRNA efficacy in the eye is not due to specific gene silencing, but is instead caused by nonspecific stimulation of the TLR3 pathway, which can reduce angiogenesis ${ }^{102}$. Although this study calls into question the nature of the anti-angiogenic effect reported in AMD clinical trials, it does not explain the therapeutic effects observed in other applications of siRNA in which appropriate controls have been performed.

Also of note is Alnylam's RSV01 formulation, which targets the nucleocapsid N gene of RSV - a major cause of respiratory illness in infants and young children. The RSV01 formulation completed Phase I trials and was found to be well tolerated in healthy adults. Since then, Phase II trials have demonstrated significant antiviral efficacy of this formulation in adults ${ }^{103}$.

\section{Future directions and conclusions}

Moving forward, we expect that synthetic nanoparticles composed of polymers, lipids, lipidoids or conjugates will have a key role in the systemic application of siRNA in the clinic. The incorporation of tissue-specific ligands into these particles may enable targeting, which will assist with in vivo biodistribution and delivery. We also anticipate that future developments will require attention to nonspecific activation of the immune system by siRNA, including TLR3 and TLR7 pathways ${ }^{102,104}$. Given the potential for nonspecific effects, it is important that the therapeutic mode of action be validated when possible, such as through direct measurement of target mRNA levels in vivo. Chemical modification of siRNA - such as 2'-O-methyl substitutions - can minimize nonspecific effects $^{40}$, and we expect that additional improvements in both activity and delivery will be mediated by direct chemical modification of the siRNA sequence.

In summary, the field of RNAi therapeutics has made significant progress since the first demonstration of gene knockdown in mammalian cells. siRNA-based formulations offer significant potential as therapeutic agents to induce the potent, persistent and specific silencing of a broad range of genetic targets. Delivery remains the most significant barrier to the widespread use of RNAi therapeutics in a clinical setting, and future work focusing on the development of safe and effective delivery materials is needed to ensure the broadest application of RNAi in the clinic. 
1. Fire, A. et al. Potent and specific genetic interference by double-stranded RNA in Caenorhabditis elegans. Nature 391, 806-811 (1998)

The first study to describe the phenomenon of RNAi.

2. Elbashir, S. M. et al. Duplexes of 21-nucleotide RNAs mediate RNA interference in cultured mammalian cells. Nature 411, 494-498 (2001)

The first study to demonstrate that exogenous siRNA is capable of sequence-specific knockdown in mammalian cells.

3. McCaffrey, A. P. et al. Gene expression: RNA interference in adult mice. Nature 418, 38-39 (2002).

Describes the first observation of sequence-specific gene silencing in mice using siRNA.

4. Morrissey, D. V. et al. Potent and persistent in vivo anti-HBV activity of chemically modified siRNAs. Nature Biotech. 23, 1002-1007 (2005).

5. Okumura, A., Pitha, P. M. \& Harty, R. N ISG 15 inhibits Ebola VP40 VLP budding in an L-domain-dependent manner by blocking Nedd4 ligase activity. Proc. Natl Acad. Sci. USA 105 3974-3979 (2008).

6. Ptasznik, A., Nakata, Y., Kalota, A., Emerson, S. G. \& Gewirtz, A. M. Short interfering RNA (siRNA) targeting the Lyn kinase induces apoptosis in primary, and drugresistant, BCR-ABL1+ leukemia cells. Nature Med. 10, 1187-1189 (2004)

7. Kim, S. H., Jeong, J. H., Lee, S. H., Kim, S. W. \& Park, T. G. Local and systemic delivery of VEGF siRNA using polyelectrolyte complex micelles for effective treatment of cancer. J. Control. Release 129, 107-116 (2008).

8. Xia, C.-F., Zhang, Y., Zhang, Y., Boado, R. \& Pardridge, W. Intravenous siRNA of brain cancer with receptor targeting and avidin-biotin technology. Pharm. Res. 24, 2309-2316 (2007).

9. Bernstein, E., Caudy, A. A., Hammond, S. M. \& Hannon, G. J. Role for a bidentate ribonuclease in the initiation step of RNA interference. Nature 409 363-366 (2001)

10. Grimm, D. et al. Fatality in mice due to oversaturation of cellular microRNA/short hairpin RNA pathways. Nature 441, 537-541 (2006).

11. Rand, T. A., Ginalski, K., Grishin, N. V. \& Wang, X. Biochemical identification of Argonaute 2 as the sole protein required for RNA-induced silencing complex activity. Proc. Natl Acad. Sci. USA 101, 14385-14389 (2004).

12. Matranga, C., Tomari, Y., Shin, C., Bartel, D. P. \& Zamore, P. D. Passenger-strand cleavage facilitates assembly of siRNA into ago2-containing RNAi enzym complexes. Cell 123, 607-620 (2005).

13. Ameres, S. L., Martinez, J. \& Schroeder, R. Molecular basis for target RNA recognition and cleavage by human RISC. Cell 130, 101-112 (2007).

14. Rand, T. A., Petersen, S., Du, F. \& Wang, X. Argonaute 2 cleaves the anti-guide strand of siRNA during RISC activation. Cell 123, 621-629 (2005).

15. Hutvagner, G. \& Zamore, P. D. A microRNA in a multiple-turnover RNAi enzyme complex. Science 297, 2056-2060 (2002)

16. Bartlett, D. W. \& Davis, M. E. Insights into the kinetics of siRNA-mediated gene silencing from live-cell and live-animal bioluminescent imaging. Nucleic Acids Res. 34, 322-333 (2006)

17. Frank-Kamenetsky, M. et al. Therapeutic RNAi targeting PCSK9 acutely lowers plasma cholesterol in rodents and LDL cholesterol in nonhuman primates. Proc. Natl Acad. Sci. USA 105, 11915-11920 (2008).

18. Sato, Y. et al. Resolution of liver cirrhosis using vitamin A-coupled liposomes to deliver siRNA against a collagen-specific chaperone. Nature Biotech. 26 431-442 (2008)

19. Song, E. et al. RNA interference targeting Fas protects mice from fulminant hepatitis. Nature Med. 9, 347-351 (2003)

20. Niu, X.-Y., Peng, Z. L., Duan, W. Q., Wang, H. \& Wang, P. Inhibition of HPV 16 E6 oncogene expression by RNA interference in vitro and in vivo. Int. J. Gynecol. Cancer 16, 743-751 (2006).

21. Halder, J. et al. Focal adhesion kinase targeting using in vivo short interfering RNA delivery in neutral liposomes for ovarian carcinoma therapy. Clin. Cancer Res. 12, 4916-4924 (2006).

22. Takeshita, F. et al. Efficient delivery of small interfering RNA to bone-metastatic tumors by using atelocollagen in vivo. Proc. Natl Acad. Sci. USA 102, 12177-12182 (2005).
23. DiFiglia, M. et al. Therapeutic silencing of mutant huntingtin with siRNA attenuates striatal and cortical neuropathology and behavioral deficits. Proc. Natl Acad. Sci. USA 104, 17204-17209 (2007).

24. de Fougerolles, A. \& Novobrantseva, T. siRNA and the lung: research tool or therapeutic drug? Curr. Opin. Pharmacol. 8, 280-285 (2008)

25. Inoue, T. et al. Modulation of scratching behavior by silencing an endogenous cyclooxygenase- 1 gene in the skin through the administration of siRNA. J. Gene Med. 9, 994-1001 (2007).

26. Fattal, E. \& Bochot, A. Ocular delivery of nucleic acids: antisense oligonucleotides, aptamers and siRNA Adv. Drug Deliv. Rev. 58, 1203-1223 (2006).

27. Palliser, D. et al. An siRNA-based microbicide protects mice from lethal herpes simplex virus 2 infection. Nature 439, 89-94 (2006).

28. Grzelinski, M. et al. RNA interference-mediated gene silencing of pleiotrophin through polyethyleniminecomplexed small interfering RNAs in vivo exerts antitumoral effects in glioblastoma xenografts. Hum. Gene Ther. 17, 751-766 (2006).

29. Bitko, V., Musiyenko, A., Shulyayeva, O. \& Barik, S Inhibition of respiratory viruses by nasally administered siRNA. Nature Med. 11, 50-55 (2005).

30. Akinc, A et al. A combinatorial library of lipid-like materials for delivery of RNAi therapeutics. Nature Biotech. 26, 561-569 (2008).

Describes the combinatorial synthesis and in vivo delivery performance of a large library of lipid-like materials in multiple animal models and species.

31. DeVincenzo, J. et al. Evaluation of the safety, tolerability and pharmacokinetics of ALN-RSV01, a novel RNAi antiviral therapeutic directed against respiratory syncytial virus (RSV). Antiviral Res. 77 225-231 (2008)

32. Li, B.-J. et al. Using siRNA in prophylactic and therapeutic regimens against SARS coronavirus in Rhesus macaque. Nature Med. 11, 944-951 (2005).

33. Alexis, F., Pridgen, E., Molnar, L. K. \& Farokhzad, O. C Factors affecting the clearance and biodistribution of polymeric nanoparticles. Mol. Pharm. 5, 505-515 (2008)

34. Scherphof, G. L. in Targeted Drug Delivery (ed. Juliano, R. L.) 285-313 (Springer, Berlin, 1991).

35. Zămecník, J., Vargovâ, L., Homola, A., Kodet, R. \& Syková, E. Extracellular matrix glycoproteins and diffusion barriers in human astrocytic tumours. Neuropathol. Appl. Neurobiol. 30, 338-350 (2004).

36. Oliveira, S., van Rooy, I., Kranenburg, O., Storm, G. \& Schiffelers, R. Fusogenic peptides enhance endosomal escape improving siRNA-induced silencing of oncogenes. Int. J. Pharm. 331, 211-214 (2007).

37. Di Guglielmo, G. M., Le Roy, C., Goodfellow, A. F. \& Wrana, J. L. Distinct endocytic pathways regulate TGF- $\beta$ receptor signalling and turnover. Nature Cell Biol. 5, 410-421 (2003)

38. Hornung, V. et al. Sequence-specific potent induction of IFN- $\alpha$ by short interfering RNA in plasmacytoid dendritic cells through TLR7. Nature Med 11 263-270 (2005)

The TLR7 pathway is identified as an important contributor to nonspecific siRNA immune activation.

39 Judge, A. D. et al. Sequence-dependent stimulation of the mammalian innate immune response by synthetic siRNA. Nature Biotech. 23, 457-462 (2005).

40. Judge, A. D., Bola, G., Lee, A. C. H. \& MacLachlan, I. Design of noninflammatory synthetic siRNA mediating potent gene silencing in vivo. Mol. Ther. 13, 494-505 (2006).

41. Vornlocher, H.-P. et al. Nuclease-resistant doublestranded RNA for RNA interference. WO2005115481 (2005)

42. Jackson, A. L. et al. Position-specific chemica modification of siRNAs reduces "off-target" transcript silencing. RNA 12, 1197-1205 (2006)

43. Bumcrot, D., Manoharan, M., Koteliansky, V. \& Sah, D. W. Y. RNAi therapeutics: a potential new class of pharmaceutical drugs. Nature Chem. Biol. 2 , 711-719 (2006).

44. Muratovska, A. ¿ Eccles, M. R. Conjugate for efficient delivery of short interfering RNA (siRNA) into mammalian cells. FEBS Lett. 558, 63-68 (2004).

45. Oishi, M., Nagasaki, Y., Itaka, K., Nishiyama, N. \& Kataoka, K. Lactosylated poly(ethylene glycol)-siRNA conjugate through acid-labile $\beta$-thiopropionate linkage to construct $\mathrm{pH}$-sensitive polyion complex micelles achieving enhanced gene silencing in hepatoma cells. J. Am. Chem. Soc. 127, 1624-1625 (2005)
46 Soutschek, J et al. Therapeutic silencing of an endogenous gene by systemic administration of modified siRNAs. Nature 432, 173-178 (2004)

47. Wolfrum, C. et al. Mechanisms and optimization of in vivo delivery of lipophilic siRNAs. Nature Biotech. 25, 1149-1157 (2007)

48. Merki, E. et al. Antisense oligonucleotide directed to human apolipoprotein B-100 reduces lipoprotein(a) levels and oxidized phospholipids on human apolipoprotein B-100 particles in lipoprotein(a) transgenic mice. Circulation 118, 743-753 (2008).

49. Isis Pharmaceuticals. Genzyme and Isis Begin Second Phase 3 Trial Of Mipomersen. Isis Pharmaceuticals web site [online], < http://ir.isispharm.com releasedetail.cfm? ReleaselD $=326694>$ (2008)

50. Torchilin, V. P. et al. Cell transfection in vitro and in vivo with nontoxic TAT peptide-liposome-DNA complexes. Proc. Natl Acad. Sci. USA 100, 1972-1977 (2003).

51. Auguste, D. T. et al. Triggered release of siRNA from poly(ethylene glycol)-protected, $\mathrm{pH}$-dependent liposomes. J. Control. Release 130, 266-274 (2008)

52. Martina, M.-S. et al. The in vitro kinetics of the interactions between PEG-ylated magnetic-fluid-loaded liposomes and macrophages. Biomaterials 28, 4143-4153 (2007).

53. Mao, S. et al. Influence of polyethylene glycol chain length on the physicochemical and biological properties of poly(ethylene imine)-graft-poly(ethylene glycol) block copolymer/siRNA polyplexes. Bioconjug. Chem. 17, 1209-1218 (2006)

54. Li, W., Huang, Z., MacKay, J. A., Grube, S. \& Szoka, F. C. Low-pH-sensitive poly(ethylene glycol) (PEG)-stabilized plasmid nanolipoparticles: effects of PEG chain length, lipid composition and assembly conditions on gene delivery. J. Gene Med. 7, 67-79 (2005).

55. Braasch, D. A. et al. Biodistribution of phosphodiester and phosphorothioate siRNA. Bioorg. Med. Chem. Lett. 14, 1139-1143 (2004).

56. Bogdanov, A. A. Merging molecular imaging and RNA interference: early experience in live animals. J. Cell Biochem. 104, 1113-1123 (2008)

57. Zimmermann, T. S. et al. RNAi-mediated gene silencing in non-human primates. Nature 441, 111-114 (2006) The first study to demonstrate sequence-specific RNAi in non-human primates.

58. Yuan, H. et al. Effects of cholesterol-tagged small interfering RNAs targeting 12/15-lipoxygenase on parameters of diabetic nephropathy in a mouse mode of type 1 diabetes. Am. J. Physiol. Renal Physiol. 295, F605-F617 (2008)

59. Rappaport, J. et al. Transport of phosphorothioate oligonucleotides in kidney: implications for molecular therapy. Kidney Int. 47, 1462-1469 (1995).

60. Santel, A et al. A novel siRNA-lipoplex technology for RNA interference in the mouse vascular endothelium. Gene Ther. 13, 1222-1234 (2006).

61. van de Water, F. M. et al. Intravenousely administered short interfering RNA accumulates in the kidney and selectively suppresses gene function in renal proximal tubules. Drug Metab. Dispos. 34, 1393-1397 (2006)

62. Takei, Y., Kadomatsu, K., Yuzawa, Y., Matsuo, S. 8 Muramatsu, T. A small interfering RNA targeting vascular endothelial growth factor as cancer therapeutics. Cancer Res. 64, 3365-3370 (2004).

63. McNamara, J. O. et al. Cell type-specific delivery of siRNAs with aptamer-siRNA chimeras. Nature Biotech 24, 1005-1015 (2006)

64. Song, E. et al. Antibody mediated in vivo delivery of small interfering RNAs via cell-surface receptors. Nature Biotech. 23, 709-717 (2005).

65. Peer, D., Park, E. J., Morishita, Y., Carman, C. V. $\delta$ Shimaoka, M. Systemic leukocyte-directed siRNA delivery revealing Cyclin D1 as an anti-inflammatory target. Science 319, 627-630 (2008).

66. Barquinero, J., Eixarch, H. \& Perez-Melgosa, M. Retroviral vectors: new applications for an old tool. Gene Ther. 11, S3-S9 (2004).

67. Vandenbroucke, R. E. et al. Prolonged gene silencing in hepatoma cells and primary hepatocytes after smal interfering RNA delivery with biodegradable poly $(\beta-$ amino esters). J. Gene Med. 10, 783-794 (2008).

68. Torchilin, V. P. Recent advances with liposomes as pharmaceutical carriers. Nature Rev. Drug Discov. 4, 145-160 (2005)

69. MacLachlan, I. in Antisense Drug Technology: Principles, Strategies and Applications. 2nd edn Ch. 9 (ed. Crooke, S. T.) 237-270 (CRC, Boca Raton, 2007).

70. Felgner, P. L. et al. Lipofection: a highly efficient, lipid-mediated DNA-transfection procedure. Proc. Natl Acad. Sci. USA 84, 7413-7417 (1987). 
71. Malone, R. W., Felgner, P. L. \& Verma, I. M. Cationic liposome-mediated RNA transfection. Proc. Natl Acad. Sci. USA 86, 6077-6081 (1989).

72. Geisbert, T. W. et al. Postexposure protection of guinea pigs against a lethal ebola virus challenge is conferred by RNA interference. J. Infect. Dis. 193 . 1650-1657 (2006).

73. Kumar, P., Lee, S. K., Shankar, P. \& Manjunath, N. A single siRNA suppresses fatal encephalitis induced by two different Flaviviruses. PLoS Med. 3, e96 (2006).

74. Li, W. \& Szoka, F. Lipid-based nanoparticles for nucleic acid delivery. Pharm. Res. 24, 438-449 (2007)

75. Wheeler, C. J. et al. A novel cationic lipid greatly enhances plasmid DNA delivery and expression in mouse lung. Proc. Natl Acad. Sci. USA 93 11454-11459 (1996).

76. Lv, H., Zhang, S., Wang, B., Cui, S. \& Yan, J. Toxicity of cationic lipids and cationic polymers in gene delivery. J. Control. Release 114, 100-109 (2006).

77. Ma, Z et al Cationic lipids enhance siRNA-mediated interferon response in mice. Biochem. Biophys. Res. Commun. 330, 755-759 (2005).

78. Akhtar, S. \& Benter, I. Toxicogenomics of non-viral drug delivery systems for RNAi: potential impact on siRNA-mediated gene silencing activity and specificity. Adv. Drug Deliv. Rev. 59, 164-182 (2007).

79. Akhtar, S. \& Benter, I. F. Nonviral delivery of synthetic siRNAs in vivo. J. Clin. Invest. 117, 3623-3632 (2007).

80. Hollins, A J., Omidi, Y, Benter I. F \& Akhtar, S. Toxicogenomics of drug delivery systems: exploiting delivery system-induced changes in target gene expression to enhance siRNA activity. J. Drug Target. 15, 83-88 (2007)

81. Rust, D. M. \& Jameson, G. The novel lipid delivery system of amphotericin B: drug profile and relevance to clinical practice. Oncol. Nurs. Forum 25, 35-48 (1998).

82. John, M. et al. Effective RNAi-mediated gene silencing without interruption of the endogenous microRNA pathway. Nature 449, 745-747 (2007). Demonstration that specific, non-viral RNAi delivery can be achieved without interruption of endogenous microRNA processing.

83. Epiphanio, S et al. Heme oxygenase- 1 is an antiinflammatory host factor that promotes murine Plasmodium liver infection. Cell Host Microbe 3, 331-338 (2008)

84. Boussif, O. et al. A versatile vector for gene and oligonucleotide transfer into cells in culture and in vivo: polyethylenimine. Proc. Natl Acad. Sci. USA 92, 7297-7301 (1995)
85. Putnam, D. Polymers for gene delivery across length scales. Nature Mater. 5, 439-451 (2006).

86. Akinc, A., Thomas, M., Klibanov, A. M. \& Langer, R. Exploring polyethylenimine-mediated DNA transfection and the proton sponge hypothesis. J. Gene Med. 7, 657-663 (2005).

87. Wang, Y., Chen, P. \& Shen, J. The development and characterization of a glutathione-sensitive cross-linked polyethylenimine gene vector. Biomaterials 27, 5292-5298 (2006)

88. Lungwitz, U., Breunig, M., Blunk, T. \& Göpferich, A. Polyethylenimine-based non-viral gene delivery systems. Eur. J. Pharm. Biopharm. 60, 247-266 (2005).

89. Zintchenko, A., Philipp, A., Dehshahri, A. \& Wagner, E. Simple modifications of branched PEI lead to highly efficient siRNA carriers with low toxicity. Bioconjug Chem. 19, 1448-1455 (2008).

90. Tan, P. H., Yang, L. C., Shih, H. C., Lan, K. C. \& Cheng, J. T. Gene knockdown with intrathecal siRNA of NMDA receptor NR2B subunit reduces formalininduced nociception in the rat. Gene Ther. 12, 59-66 (2004)

91. Urban-Klein, B., Werth, S., Abuharbeid, S., Czubayko, F. \& Aigner, A. RNAi-mediated gene-targeting through systemic application of polyethylenimine (PEI)complexed siRNA in vivo. Gene Ther. 12, 461-466 (2004)

92. Kichler, A. Gene transfer with modified polyethylenimines. J. Gene Med. 6. S3-S10 (2004).

93. Kircheis, R., Wightman, L. \& Wagner, E. Design and gene delivery activity of modified polyethylenimines. Adv. Drug Deliv. Rev. 53, 341-358 (2001).

94. Richards Grayson, A., Doody, A. \& Putnam, D. Biophysical and structural characterization of polyethylenimine-mediated siRNA delivery in vitro. Pharm. Res. 23, 1868-1876 (2006).

95. Thomas, M. et al. Full deacylation of polyethylenimine dramatically boosts its gene delivery efficiency and specificity to mouse lung. Proc. Natl Acad. Sci. USA 102, 5679-5684 (2005).

96. Werth, S. et al. A low molecular weight fraction of polyethylenimine (PEI) displays increased transfection efficiency of DNA and siRNA in fresh or lyophilized complexes. J. Control. Release 112, 257-270 (2006)

97. Hu-Lieskovan, S., Heidel, J. D., Bartlett, D. W., Davis, M. E. \& Triche, T. J. Sequence-specific knockdown of EWS-FLI1 by targeted, nonviral delivery of small interfering RNA inhibits tumor growth in a murine model of metastatic Ewing's sarcoma. Cancer Res. 65, 8984-8992 (2005).
98. Heidel, J. D et al. Administration in non-human primates of escalating intravenous doses of targeted nanoparticles containing ribonucleotide reductase subunit M2 siRNA. Proc. Natl Acad. Sci. USA 104. 5715-5721 (2007)

99 Bartlett, D. W., Su, H., Hildebrandt, I. J., Weber, W. A ¿ Davis, M. E. Impact of tumor-specific targeting on the biodistribution and efficacy of siRNA nanoparticles measured by multimodality in vivo imaging. Proc. Natl Acad. Sci. USA 104, 15549-15554 (2007).

100. Bartlett, D. W. \& Davis, M. E. Impact of tumor-specific targeting and dosing schedule on tumor growth inhibition after intravenous administration of siRNAcontaining nanoparticles. Biotechnol. Bioeng. 99 975-985 (2008).

101. Rozema, D. B. et al. Dynamic PolyConjugates for targeted in vivo delivery of siRNA to hepatocytes. Proc. Natl Acad. Sci. USA 104, 12982-12987 (2007)

102. Kleinman, M. E. et al. Sequence- and targetindependent angiogenesis suppression by siRNA via TLR3. Nature 452, 591-597 (2008). Demonstration that the anti-angiogenic effects seen upon siRNA delivery to the eye can be nonspecific and caused by TLR3 immune activation.

103. Alnylam Pharmaceuticals. Alnylam Achieves First Human Proof of Concept for an RNAi Therapeutic with GEMINI study. Alnylam Pharmaceuticals web site [online] < http://phx.corporate-ir.net/phoenix. zhtml? $\mathrm{c}=148005 \& \mathrm{p}=$ irol-newsArticle $2 \& \mid \mathrm{D}=11139$ 37 \&highlight $=>$ (2008).

104. Robbins, M. et al. Misinterpreting the therapeutic effects of siRNA caused by immune stimulation Hum. Gene Ther. 19 Aug 2008 (doi 10.1089/ hgt.2008.131)

105. Zhang, Y. et al. Engineering mucosal RNA interference in vivo. Mol. Ther. 14, 336-342 (2006)

106. Arnold, A.-S. et al. Specific $\beta 1$-adrenergic receptor silencing with small interfering RNA lowers high blood pressure and improves cardiac function in myocardial ischemia. J. Hypertens. 25, 197-205 (2007).

\section{Acknowledgements}

The authors would like to thank A. de Fougerolles, R. Alvarez and $\mathrm{E}$. Deguns for their advice and assistance in the preparation of the manuscript.

DATABASES

UniProtKB: http://www.uniprot.org

PCSK9 | ILR3 | ILR7|VEGE|VEGFR

ALL LINKS ARE ACTIVE IN THE ONLINE PDF 
CORRIGENDUM

Knocking down barriers: advances in siRNA delivery

Kathryn A. Whitehead, Robert Langer \& Daniel G. Anderson

Nature Reviews Drug Discovery 8, 129-138 (2009) | doi:10.1038/nrd2742

On page 136 in Table 3, the clinical trial for acute renal failure has been attributed to Quark/Pfizer when it should be attributed to Quark alone. In addition, the authors would like to clarify that, following a Phase I/II trial in patients with wet age-related macular degeneration, the siRNA drug candidate PF-4523655 (RTP801i-14) is now being studied in a Phase II trial for diabetic macular oedema conducted by Pfizer in collaboration with Quark. 